\title{
How do I treat when there is no treatment?
}

\author{
Debra S. Regier ${ }^{\mathrm{a}, *}$, E. Hallie Andrew ${ }^{\mathrm{a}}$, Miriam Bloom ${ }^{\mathrm{b}}$ and Deborah Fisher ${ }^{\mathrm{c}}$ \\ ${ }^{a}$ Rare Disease Institute, Children's National Hospital, Washington DC, USA \\ ${ }^{\mathrm{b}}$ Hospitalist Division, Children's National Hospital, Washington DC, USA \\ 'PANDA Palliative Care Team, Children's National Hospital, Washington DC, USA
}

\begin{abstract}
The majority of children in hospice care do not have cancer, but many have rare diseases. Thus, it is essential that rare disease specialists have a functioning knowledge of the palliative care system and its role in meeting the unique needs of pediatric patients with genetic disorders or inborn errors of metabolism. Creating a team of providers that both understands the natural history of the specific rare disease and truly understands the principles of palliative care leads to optimal outcomes for patients. Here, we discuss the importance of genetic counseling and describe the basic tenants of palliative care. By approaching this topic from a rare disease perspective, we discuss the unique features of improving quality of life for patients and families. In addition, we discuss the role of self-care in preventing care provider burnout of families and medical providers. The thoughtful approach to palliation in rare diseases is essential to optimize the experience for all participants in a patient's shortened life.
\end{abstract}

Keywords: Rare disease, hospice, palliative care, symptom management, quality of life

It is from knowledge tempered with humility that I know I will simultaneously be right and wrong in my impressions, yet I share my experiences sitting in the sacred place of telling a family we know why their child is suffering from disease, but have no way to stop it. This paper is dedicated to the families that I have learned from-those that have been honest and courageous enough to tell me what I did right (and more importantly wrong!). Those families who took the initiative to ask me when I didn't know how to tell them the end was near. I do not live in this sacred space alone. Instead, I am surrounded by an amazing community that finds a way to treat when there is no treatment. Our goal in this manuscript is to equip care providers so that they can sit with families at funerals with no regrets.

\section{Introduction}

With more than 7,000 rare diseases and under 700 treatments, the most common, single treatment prescribed is not a medication or gene therapy; instead, it is supportive therapies to improve overall quality of life. Rather than saying "there is no treatment", providers instead say, "there is no cure, but we have so many ways to optimize life to its fullest." While often considered a need for oncology patients, palliative care is important for children and adults with life-shortening rare disorders. Unlike the adult palliative care population, only $30-40 \%$ of the patients in pediatric palliative care have cancer [5]. In a care center in British Columbia, the leading cause for palliative care referral was central nervous system conditions $(27 \%)$, followed by chromosomal anomalies $(20 \%)$, and then cancer $(16 \%)$. Metabolic or biochemical abnormalities (14\%), cardiopulmonary (10\%), and immunologic or infectious etiologies were less commonly seen in the patient population (1\%) [25]. Similar findings have been seen in the United States $[5,7]$. Thus, the expected patient participants in pediatric palliative care are less

\footnotetext{
${ }^{*}$ Corresponding author: Dr. Regier, Rare Disease Institute, Children's National Hospital, Washington DC, USA. E-mail: DRegier@childrensnational.org.
} 
commonly oncological patients and more commonly those diagnosed by the genetics and metabolism community.

For some diagnoses, the approach is to optimize supportive therapies and interventions for a child or adult with rare disorders. This approach has been well characterized in treatment guidelines. For example, in many metabolic disorders there is no cure or medication treatment, but extreme dietary interventions can improve outcomes and quality of life for the patient [13].

At the end of many of these potentially distressing conversations is the hope of treatments in the "pipeline" and helping the patient enroll in research trials. The more difficult conversation for practitioners is not that there is no cure or treatment, but that we cannot prevent early death. The provider has to balance hope for a treatment in the future with the reality of the life expectancy for the patient. In this challenging situation, setting reasonable expectations requires a hopeful honesty.

Familiar with the natural history of the rare diseases, it is often the geneticist who initiates the palliative care referral. While nearly non-existent in the genetics and metabolism field, lessons learned from oncology show that inclusion of a palliative care physician and/or team is helpful for the family in comparison to care by the oncologist alone $[12,14]$. In addition, the palliative team is often more comfortable and experienced with balancing hope and planning for an early death with families. These difficult discussions are truly ongoing conversations, and do not occur in just one meeting. The long-term progression towards acceptance is important for both families and care providers.

\section{Palliative approaches can be customized to unique diagnoses}

The holistic approach to palliative care developed with oncology patients can also be effective for children with rare disorders, with some caveats. Pediatric palliative care is a holistic approach that prevents, identifies, and treats all types of physical and non-physical suffering in children with a serious illness such as a genetic disorder [7]. The traditional hospice program for cancer patients can more easily prognosticate a six-month survival trajectory and focus on end of life and "dying well." In contrast, the trajectory of genetic and metabolic disorders is much less focused. Fragile patients with chronic, slowly progressive disease may succumb to their illness as a result of an acute event, such as infection. A more contemporary model for pediatric palliative care for genetic and metabolic disorders is required. This would include initiating care at the time of diagnosis of a lifelimiting illness with a focus on aggressive symptom management in order to optimize quality of life and achieve individualized goals. These goals are best achieved through ongoing, fluid conversations between families and caregivers. The patient, if able, along with the family shares their wishes and hopes. The pediatric palliative care team offers therapeutic presence to facilitate understanding and acclimation to the "new normal" with adjusted goals and expectations. In addition to easing comfort and increasing the potential to achieve goals, optimizing quality of life through aggressive symptom management is also necessary to allow for higher order tasks such as advanced care planning, memory making, and anticipatory bereavement.

Even though the lifespan may be shortened, the time spent can be very meaningful and precious. A position paper from the American Academy of Pediatrics (AAP) states that pediatric palliative care should emphasize quality rather than quantity by focusing on adding "life to the child's years, not simply years to the child's life" [1]. An instructive video prepared for the AAP illustrating this approach in a rare disease patient is Cameron's Arc. Open communication and clear goals for care planning and delivery are also important to avoid conflict and misunderstanding $[2,6]$. Ongoing communication about what to expect in the final days and weeks is critical in helping families through this difficult time. In a landmark qualitative study, bereaved parents shared that pain, dyspnea, anorexia, and fatigue were the four most bothersome symptoms in their dying child [26]. 


\section{Symptoms can be managed effectively}

Another role of the geneticist is to understand and treat the pain associated with a given disease such as visceral, neurologic, or bone-mediated pain. By understanding the underlying etiology of disease, effective first- and second-line pain management plans can often be optimized for each patient. For example, the pain profiles in mucopolysaccharidoses and sphingolipidoses have been characterized and some disorders even have pain management treatment guidelines [4]. For ultra-rare disorders there are often no guidelines or case reports to follow. Thus, pain and distressing symptoms must be addressed on a case by case basis by providers expert in diverse management strategies.

Pain assessment in the neurologically impaired child is a challenge. As Hauer discusses in her seminal work, identifying pain signs and symptoms is the first step towards managing pain [9]. While self-report of pain is the gold standard, using validated assessment tools such as a behavioral assessment tool is necessary in young or nonverbal children. Once the level of pain has been identified, it is then necessary to define pain versus agitation versus visceral hyperalgesia [9]. Thus, pain management approaches for concerns for visceral hyperalgesia could include anti-epileptic medications, tricyclic antidepressants, $\alpha$ agonists, to opioids [9]. In addition, hip subluxation, spasticity, pressure ulcers and gastroesophageal reflux are common sources of pain. These require specific managements based on their etiologies. Thus, a careful assessment of pain level and suspected pain source will directly alter medication management.

\subsection{Sleep management}

Many genetic and metabolic disorders have unique sleep patterns, leading to dysautonomia that can worsen towards the end of life. Anticipating disordered sleep can allow for changes in dose or timing of medications and may also allow for proactive adjustment in overnight support for families caring for their child at home. Caregiver fatigue can be a major issue in children with disordered sleep.

\subsection{Dyspnea}

Dyspnea is a common terminal finding [26]. The sensation of breathlessness can be assessed using the Dalhousie Dyspnea scale [21] if the child is older and verbal; however, for non-verbal children, the clinician must rely on signs and symptoms of tachypnea, and increased work of breathing such as use of accessory muscles. Treatments for dyspnea are straightforward and include flowing air, oxygen support, and pain medications, but these interventions may represent an increase in the level of care and should be discussed with the family well before they are needed [19]. Metabolic and genetics patients can have alterations in metabolites that lead to changes in respiratory state. For example, a patient with a known organic acidemia with disease progression can have worsening respiratory status from acidosis and increased work of breathing from systemic decline.

\subsection{Neurological symptoms should be attended}

Management of neurological symptoms is crucial for many patients with inborn errors of metabolism or other genetic disorders. In disorders with brain malformations, metabolic disorders, or storage disorders, the neurological symptoms can progress rapidly or more slowly over time. Aggressive treatment of seizures to prevent distress to the patient and family can be very important in improving the quality of life. Other neurological issues that commonly arise and need to be addressed include: spasticity, dystonia, unusual movements, and progressive immobility. Neuro-irritability can be associated with many disorders and is particularly distressful to families and care providers. Managing neurologic 
symptoms of genetic and metabolic diseases is complex and is often best handled by a team including neurologists, geneticists, and physiatrists.

\subsection{Feeding issues}

Feeding issues are also complex in children with genetic or metabolic disorders. Some lose ability to eat by mouth due to muscular or coordination causes, while those with storage disorders tend to have incoordination and weakness leading to aspiration events. In addition, the underlying metabolic disorder and the degree of physical activity may make the caloric needs for the child in palliative care different from that of a typically developed child of the same age. Caloric requirements may range from $50 \%$ of age-matched typically developed children to over $150 \%$ of expected as shown by metabolic calorimetry (personal experience).

\subsection{Temperature regulation}

Many children with mitochondrial disorders (either primary or secondary to other metabolic or genetic syndromes) can have dysregulation of temperature. Little has been published in the medical literature, however, parents indicate this is a concern that they would like to treat and do so environmentally with attention to the hands and feet to prevent injury. Metabolic physicians often comment on this concern and identify symptomatic plans to reduce suffering (author's experience and personal communication from Kimberly Chapman, MD PhD and Rebecca Ganetzky, MD PhD).

\subsection{Progression to end stage disease}

When the underlying disorder progresses over months or years, it is important to recognize that the clinical decline impacts nearly every physiologic system. For example, in the case of progressive weakness, eventual respiratory failure is often preceded by recurrent infection, aspiration, upper airway obstruction, and lack of central control of breathing. Likewise, worsening autonomic dysfunction leads to gradual impairment of gastrointestinal function, whereby constipation leads to generalized dysmotility and finally intestinal failure, and impaired urologic function leads to a neurogenic bladder. As a child becomes less mobile, close screening for skin and pressure ulcers is also vital to minimizing discomfort. Maintaining the child's quality of life requires attention to all aspects of care. Although there may be a myriad of non-invasive and invasive interventions that can be implemented, symptomatic management may be the best path for the child and the family. These issues are best managed by ongoing discussion of care goals between healthcare providers and the family to make the best possible decision for each individual child.

A holistic approach with a combination of aggressive palliation, access to hospice care, and the expertise of a geneticist with an understanding of disease progression and prediction of future needs is the ideal care delivery model for a child with a genetic or metabolic disorder. This approach is optimized when a geneticist is part of the palliative care team.

\section{The geneticist and the diagnostic odyssey}

Patients with suspected genetic conditions and their families have different needs that must be met by a variety of qualified medical providers. In addition to medical management such as medication changes, screenings, or quality of life care, many families struggle with obtaining an accurate diagnosis. These patients may be referred to the genetics clinic as part of the "diagnostic odyssey". Even after obtaining a diagnosis for their child parents have many questions. The first is often, "Why did this 
happen?" Genetic testing may provide the answer. When test results are diagnostic, genetic counselors can help families understand the mechanism of disease and the spectrum of impact this may mean for the child's health and development.

The second question quickly follows is, "Is this my fault?" Parents feel a deep responsibility for their children, not only in being able to care and provide for them, but also in what they may have unknowingly passed to them through their DNA. Conversations about inheritance and "passing down" genetic mutations can bring up feelings of guilt or confusion. It is important for parents to hear that there is nothing they did or did not do that caused the genetic problem in their child and that none of us has control over which genes we pass our children. It is also critical to allow time for parents to process their feelings and come to terms with what they have heard. Interestingly, many parents find relief of guilt whether results of testing are positive or negative [3, 8, 15, 22]. The third question is "What does the future hold?" The genetic counselor and geneticist are empowered to work together with all members of the child's care team to determine a specific care plan and expected prognosis based on genetic testing results.

Finally, families often have questions about recurrence risk, either for existing family members or for future pregnancies. The genetic counselor can often provide precise information regarding risk for other family members, discuss reproductive options, and facilitate referrals to maternal fetal medicine providers.

Genetic counselors are equal part genetics expert and counselor. The psychosocial impacts of these questions and the resulting conversations are complex, challenging, and can be life-changing. In addition to having highly specialized training in genetics, they are well trained in psychosocial counseling techniques to facilitate the difficult conversations and strong feelings that surround the diagnostic odyssey.

\section{Quality of life can be optimized}

As we have discussed, improving the quality of life, regardless of its length, is the goal for all of the team. To this end, leveraging community-based services, supports, and resources is essential. From programs that help with rent and mortgage costs, to meal vouchers, and teachers who ensure a child is included regardless of their ability to fully participate, the greater community should be leveraged early and often to support families. In our group, we initially viewed this leveraging of services as a "service" to the child, but soon found that it was also the ideal opportunity for the community to understand rare disease, the need for treatments, and to affirm altruism within the larger community. Often families with rare diseases feel isolated or "unseen". Leveraging community involvement made families feel recognized and cared for in unexpected ways by community members.

One important way for families to feel accepted and loved is through the Make-A-Wish program. While traditionally thought of as a program for children with cancer, the program has always strived to provide a Wish for every eligible child. Recent studies show a reduction in unplanned hospitalizations and improved quality of life in children who received a Wish [20,24]. In response to feedback from the rare disease community, the national office of Make-A-Wish has created a list of genetic and metabolic diagnoses that will automatically qualify a child for a Wish and launched outreach to hospice programs to enable more eligible children with genetic and metabolic disorders to receive a Wish. A Wish is not only fun for the family, but gives them an opportunity to live life without the focus on their rare disease for a few days. They get to create their own destiny; meet their hero; or make the lives of others better. In degenerative conditions it is important to identify the right time for a Wish. It should be early enough in the illness that the child and family can fully participate. Early conversations with the local Make-A-Wish organization are important to find the right time and the right Wish for any child. 
The religious or spiritual beliefs of a family are an important component of the entire palliative care process. Understanding the family's beliefs and the community-based supports stemming from their spiritual community are an important mechanism to improve quality of life for the patient and family [10]. For providers who do not have a spiritual or faith base, this can be a difficult discussion and one may find a family and provider talking past one another. It is important that providers are comfortable initiating spiritual conversations, or helping to identify spiritual resources for families that may include the hospital chaplain, a colleague with a similar faith base, or other outside support mechanisms [18].

Regardless of the location of the family, exploration of existing community resources to optimize access to community programs is essential. Whether through a spiritual or faith-based organization, social worker, community partnership, school system, health care system, or non-profit organizations, ensuring the family has community-based support helps them to feel connected and supported.

\section{The team must also care for itself}

It can be hard for geneticists and team members to find the way to emotionally process giving a difficult or terminal diagnosis, or a diagnosis for which there is not yet an effective treatment. Ongoing exposure to suffering contributes to provider stress and may lead to compassion fatigue and burnout [16]. As a professional community, we need to accept these difficulties and acknowledge the stress and distress this can bring to all of us. In the same way that we ask families to mourn their children, we need to create spaces to mourn our own losses-both personal and professional. By caring for ourselves, we avoid compassion fatigue, promote resiliency, and enhance what we do have to offer, excellent care for our patients $[11,23]$. The first step in self-care is awareness of the risk factors and realizing that the level of stress is commensurate with the intensity, duration, and proximity of exposure [17].

Seasoned geneticists know the experience of caring for a child from birth through their death, an experience that is both satisfying and gut-wrenching. The experiences of giving difficult diagnoses and initiating palliative care may cause stress, but many providers also feel that being part of this experience provides satisfaction and adds meaning to their work.

In addition to caring for families, we must also care for ourselves and for each other. Whether it is acknowledging that we as providers have "lost" a patient, or creating a place or time to remember them, or remembering a patient's name when others have forgotten, we live in the dichotomy of grief and joy for a life well lived.

\section{Acknowledgments}

We would like to thank Miss Nancy Cheng for editorial assistance with this manuscript.

\section{References}

[1] American Academy of Pediatrics. Committee on Bioethics and Committee on Hospital Care. Palliative care for children, Pediatrics 106 (2000), 351-357.

[2] Cameron's Arc: Creating a Full Life Teaching and Resource Guide with DVD American Academy of Pediatrics, Itasca, Illinois, 2007.

[3] A. Chapple, C. May and P. Campion, Parental guilt: The part played by the clinical geneticist, J Genet Couns 4 (1995), 179-191.

[4] S. Congedi, M. Orzalesi, C. Di Pede and F. Benini, Pain in Mucopolysaccharidoses: Analysis of the Problem and Possible Treatments, Int J Mol Sci 19 (2018).

[5] C. Feudtner, T.I. Kang, K.R. Hexem, S.J. Friedrichsdorf, K. Osenga, H. Siden, S.E. Friebert, R.M. Hays, V. Dussel and J. Wolfe, Pediatric palliative care patients: A prospective multicenter cohort study, Pediatrics 127 (2011), 1094-1101. 
[6] M.J. Field, R.E. Behrman and Institute of Medicine (U.S.). Committee on Palliative and End-of-Life Care for Children and Their Families., When children die : Improving palliative and end-of-life care for children and their families, National Academy Press, Washington, D.C., 2003.

[7] S.E. Friebert and C. Williams, NHPCO's Facts and Figures: Pediatric Palliative \& Hospice Care in America, in, National Hospice and Palliative Care Organization, Alexandria, VA, 2015.

[8] E. Giarelli and M. Reiff, Mothers' appreciation of chromosomal microarray analysis for autism spectrum disorder, J Spec Pediatr Nurs 20 (2015), 244-258.

[9] J. Hauer and A.J. Houtrow, Pain Assessment and Treatment in Children With Significant Impairment of the Central Nervous System, Pediatrics 139 (2017).

[10] B.P. Himelstein, J.M. Hilden, A.M. Boldt and D. Weissman, Pediatric palliative care, N Engl J Med 350 (2004), 1752-1762.

[11] S.M. Kase, E.D. Waldman and A.S. Weintraub, A cross-sectional pilot study of compassion fatigue, burnout, and compassion satisfaction in pediatric palliative care providers in the United States, Palliat Support Care (2018), 1-7.

[12] E.C. Kaye, S. Friebert and J.N. Baker, Early Integration of Palliative Care for Children with High-Risk Cancer and Their Families, Pediatr Blood Cancer 63 (2016), 593-597.

[13] P. Kruszka and D. Regier, Inborn Errors of Metabolism: From Preconception to Adulthood, Am Fam Physician 99 (2019), 25-32.

[14] D.R. Levine, B.N. Mandrell, A. Sykes, M. Pritchard, D. Gibson, H.J. Symons, D. Wendler and J.N. Baker, Patients' and Parents' Needs, Attitudes, and Perceptions About Early Palliative Care Integration in Pediatric Oncology, JAMA Oncol 3 (2017), 1214-1220.

[15] N.L. Makela, P.H. Birch, J.M. Friedman and C.A. Marra, Parental perceived value of a diagnosis for intellectual disability (ID): A qualitative comparison of families with and without a diagnosis for their child's ID, Am J Med Genet A 149A (2009), 2393-2402.

[16] P. Meadors and A. Lamson, Compassion fatigue and secondary traumatization: Provider self care on intensive care units for children, J Pediatr Health Care 22 (2008), 24-34.

[17] P. Meadors, A. Lamson, M. Swanson, M. White and N. Sira, Secondary traumatization in pediatric healthcare providers: Compassion fatigue, burnout, and secondary traumatic stress, Omega (Westport) 60 (2009), 103-128.

[18] C.J. Morgenweck, Spirituality at the end of life, Curr Opin Anaesthesiol 31 (2018), 185-189.

[19] M. O’Nelll Hunt, B. McCrate Protus, J. Penfiled Winters and D.C. Parker, Pediatric Palliatve Care Consultant, HospiScript, Montgomery, AL, 2014.

[20] A.D. Patel, P. Glynn, A.M. Falke, M. Reynolds, R. Hoyt, A. Hoynes, M. Moore-Clingenpeel, A. Salvator and J.J. Moreland, Impact of a Make-A-Wish experience on healthcare utilization, Pediatr Res (2018).

[21] P.T. Pianosi, M. Huebner, Z. Zhang, A. Turchetta and P.J. McGrath, Dalhousie Pictorial Scales Measuring Dyspnea and Perceived Exertion during Exercise for Children and Adolescents, Ann Am Thorac Soc 12 (2015), 718-726.

[22] M. Reiff, E. Bugos, E. Giarelli, B.A. Bernhardt, N.B. Spinner, P.L. Sankar and S. Mulchandani, "Set in Stone" or "Ray of Hope": Parents' Beliefs About Cause and Prognosis After Genomic Testing of Children Diagnosed with ASD, J Autism Dev Disord 47 (2017), 1453-1463.

[23] N. Sanso, L. Galiana, A. Oliver, A. Pascual, S. Sinclair and E. Benito, Palliative Care Professionals' Inner Life: Exploring the Relationships Among Awareness, Self-Care, and Compassion Satisfaction and Fatigue, Burnout, and Coping With Death, J Pain Symptom Manage 50 (2015), 200-207.

[24] A. Shoshani, K. Mifano and J. Czamanski-Cohen, The effects of the Make a Wish intervention on psychiatric symptoms and health-related quality of life of children with cancer: A randomised controlled trial, Qual Life Res 25 (2016), 1209-1218.

[25] H. Siden, Pediatric Palliative Care for Children with Progressive Non-Malignant Diseases, Children (Basel) 5 (2018).

[26] J. Wolfe, H.E. Grier, N. Klar, S.B. Levin, J.M. Ellenbogen, S. Salem-Schatz, E.J. Emanuel and J.C. Weeks, Symptoms and suffering at the end of life in children with cancer, N Engl J Med 342 (2000), 326-333. 